\title{
Characterization and compensation of thermo-elastic instability of SWARM optical bench on Micro Advanced Stellar Compass attitude observations
}

Herceg, Matija; Jørgensen, Peter Siegbjørn; Jørgensen, John Leif

\section{Published in:}

Acta Astronautica

Link to article, DOI:

10.1016/j.actaastro.2017.04.018

Publication date:

2017

Document Version

Publisher's PDF, also known as Version of record

Link back to DTU Orbit

Citation (APA):

Herceg, M., Jørgensen, P. S., \& Jørgensen, J. L. (2017). Characterization and compensation of thermo-elastic instability of SWARM optical bench on Micro Advanced Stellar Compass attitude observations. Acta Astronautica, 137, 205-213. https://doi.org/10.1016/j.actaastro.2017.04.018

\section{General rights}

Copyright and moral rights for the publications made accessible in the public portal are retained by the authors and/or other copyright owners and it is a condition of accessing publications that users recognise and abide by the legal requirements associated with these rights.

- Users may download and print one copy of any publication from the public portal for the purpose of private study or research.

- You may not further distribute the material or use it for any profit-making activity or commercial gain

- You may freely distribute the URL identifying the publication in the public portal 


\title{
Characterization and compensation of thermo-elastic instability of SWARM optical bench on micro Advanced Stellar Compass attitude observations
}

\author{
M. Herceg*, P.S. Jørgensen, J.L. Jørgensen \\ DTU Space, Denmark Technical University, Denmark
}

\section{A R T I C L E I N F O}

\section{Keywords:}

Spacecraft attitude

Star tracker

Optical bench

\begin{abstract}
A B S T R A C T
Launched into orbit on November 22, 2013, the Swarm constellation of three satellites precisely measures magnetic signal of the Earth. To ensure the high accuracy of magnetic observation by vector magnetometer (VFM), its inertial attitude is precisely determined by $\mu$ ASC (micro Advanced Stellar Compass). Each of the three Swarm satellites is equipped with three $\mu$ ASC Camera Head Units (CHU) mounted on a common optical bench $(\mathrm{OB})$, which has a purpose of transference of the attitude from the star trackers to the magnetometer measurements. Although substantial pre-launch analyses were made to maximize thermal and mechanical stability of the $\mathrm{OB}$, significant signal with thermal signature is discovered when comparing relative attitude between the three CHU's (Inter Boresight Angle, IBA). These misalignments between CHU's, and consequently geomagnetic reference frame, are found to be correlated with the period of angle between Swarm orbital plane and the Sun (ca. 267 days), which suggests sensitivity of optical bench system on temperature variation.

In this paper, we investigate the propagation of thermal effects into the $\mu \mathrm{ASC}$ attitude observations and demonstrate how thermally induced attitude variation can be predicted and corrected in the Swarm data processing. The results after applying thermal corrections show decrease in IBA RMS from 6.41 to 2.58". The model significantly improves attitude determination which, after correction, meets the requirements of Swarm satellite mission. This study demonstrates the importance of the OB pre-launch analysis to ensure minimum thermal gradient on satellite optical system and therefore maximum attitude accuracy.
\end{abstract}

\section{Introduction}

The objective of the Swarm mission is to map the magnetic field of the Earth by a constellation of three satellites, two of them at a lower altitude and one at a higher. Swarm satellites precisely measure the magnetic signal of the Earth using a scalar (ASM) and a vector magnetometer (VFM). The ASM measures the magnetic field strength, which is used in calibration of the VFM to maintain the absolute accuracy during the mission. The VFM measures the magnetic field vector, whose inertial attitude is determined by the $\mu$ ASC (micro Advanced Stellar Compass) with high accuracy. The $\mu$ ASC is a fully autonomous, internally redundant star tracker. Three of the $\mu$ ASC, together with the VFM instrument, are mounted on the Swarm optical bench (OB) to provide correct orientation of the VFM and high accuracy of measured magnetic field components.

Considering that orientation of the Swarm satellite can be determined from both magnetic field measurements and star tracker images, independent measurement can be compared, and ideally, should agree. As addressed in [1], Swarm satellites exhibit consistent misalignments between the stellar and geomagnetic reference frames. The author correlates these misalignments, among other possible causes, with deficiencies in the software carrying out the star camera aberration correction, while completely disregarding any sort of thermally-induced mechanical instability. To analyze possible causes of these misalignments, a detailed study was performed on relative orientation of the star cameras and its correlation with stellar aberration and thermal variation of optical bench system.

The main objective of this paper is to research and explain the periodical variations between the Swarm camera head units (i.e. misalignment between the camera and geomagnetic reference frame) and to present a solution for its correction in the Swarm data processing.

\subsection{Micro Advanced Stellar Compass}

The Advanced Stellar Compass (ASC) and $\mu$ ASC were pioneers of the fully autonomous star trackers. Designed and produced by the Measurement and Instrumentation section at DTU Space department

\footnotetext{
* Corresponding author.

E-mail addresses: mher@space.dtu.dk (M. Herceg), psj@space.dtu.dk (P.S. Jørgensen), jlj@space.dtu.dk (J.L. Jørgensen).
} 
(Denmark Technical University), the $\mu \mathrm{ASC}$ is to date one of the most successful star tracker worldwide. $\mu$ ASC autonomously calculates attitude based on all bright stars in the Field of View (FOV) of the CHUs using cold/hot redundant Digital Processing Unit (DPU). Running a single CHU, $\mu$ ASC can provide 22 true solutions per second, with absolute accuracy of $<1^{\prime \prime}$ [2]. The instruments have been operating on many satellite missions, and even though they were subjected to very different orbital and thermal environment, not a single hardware or functional failure has ever occurred.

\subsection{Swarm optical bench}

The Swarm optical bench is an ultra-stable silicon carbide-carbon fiber compound structure installed on a deployable conical tube of square cross section [3]. Having the main purpose of transferring the attitude that is precisely determined by star trackers to the magnetometer field components, the $\mathrm{OB}$ serves as a highly thermal and mechanical stable platform. Therefore, prior to launch, an exhaustive thermo-elastic and thermo-mechanical analysis were carried out to ensure thermal stability. For precise monitoring of the optical bench thermal state, several thermistors are mounted on different parts of Deployable Boom Assembly (DBA). There are 4 triplets of thermistors (summarized in Table 1) where each triplet measures very similar temperatures. Thermistors on Swarm satellite OB used in the modelling are shown on Fig. 1, where $T_{1}$ is mounted on CHU CCD sensor, $T_{2}$ represents thermistor THT00029 on Optical bench Boom I/F and $T_{3}$ represents thermistor THT00032 mounted on Optical Bench next to SiC-cube.

\subsection{Swarm attitude observations}

The prime instrument of the Swarm mission is the VFM, which is mounted on the spacecraft boom. The VFM system consists of a DPU and a Compact Spherical Coil (CSC) vector magnetometer sensor mounted on a stable OB (Fig. 1). On each Swarm satellite, the three $\mathrm{CHU}$ are placed on the $\mathrm{OB}$ such that the angles between the boresights, referred to as Inter-Boresight Angles (IBA), are around $90^{\circ}$ (Fig. 2). This is to ensure that only one CHU may be affected by intrusion of big and bright objects, such as the Sun or Moon, in the field of view at any given time, allowing attitude solution availability throughout the entire mission. Each CHU mounted on Swarm satellites is observing the attitude at a rate of $1 \mathrm{~Hz}$, obtaining the precise orientation of the $\mathrm{CHU}$ relative to the J2000.0 Earth-centered inertial coordinate frame. In this study, the orientations of the three CHU's are compared by calculating and analyzing the IBA between CHU pairs (Fig. 2). There are three IBA's defined: IBA of CHU pair 1 (angle between Z-axis of CHU A and Z-axis of CHU B); IBA of CHU pair 2 (angle between Z-axis of CHU A and Z-axis of CHU C); and IBA of CHU pair 3 (angle between Z-axis of CHU B and Z-axis of CHU C).

Table 1

Summary of the thermistors mounted on Swarm deployable boom.

\begin{tabular}{lll}
\hline Thermistor name & $\begin{array}{l}\text { Thermistor } \\
\text { code } 1\end{array}$ & Thermistor name 2 \\
& THT00028 & TCL_2_ANY_13A_HINGEHNR \\
Hinge & THT00056 & TCL_4_ANY_13B_HINGEHNR \\
& THT00086 & TCL_6_ANY_13C_HINGEHNR \\
Optical bench Boom I/F & THT00029 $\left(\mathrm{T}_{2}\right)$ & TCL_2_ANG_14A_OBBOOMIF \\
& THT00057 & TCL_4_ANG_14B_OBBOOMIF \\
& THT00087 & TCL_6_ANG_14C_OBBOOMIF \\
Absolute Scalar & THT00031 & TCL_2_ANG_16A_ASMS \\
Magnetometers & THT00059 & TCL_4_ANG_16B_ASMS \\
(ASMS) & THT00089 & TCL_6_ANG_16C_ASMS \\
Optical Bench STRH & THT00032 $\left(\mathrm{T}_{3}\right)$ & TCL_2_ANG_17A_OBSUBSY \\
& THT00060 & TCL_4_ANG_17B_OBSUBSY \\
& THT00090 & TCL_6_ANG_17C_OBSUBSYS \\
\hline
\end{tabular}

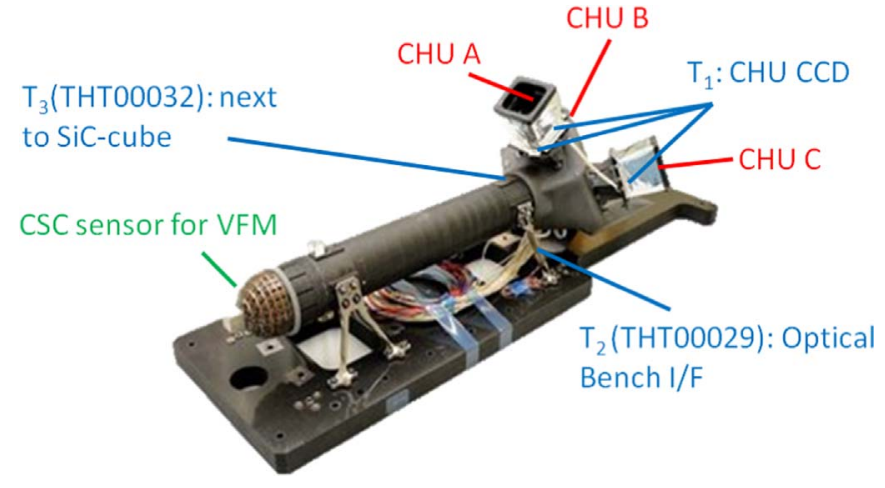

Fig. 1. Thermistors on Swarm satellite optical bench.

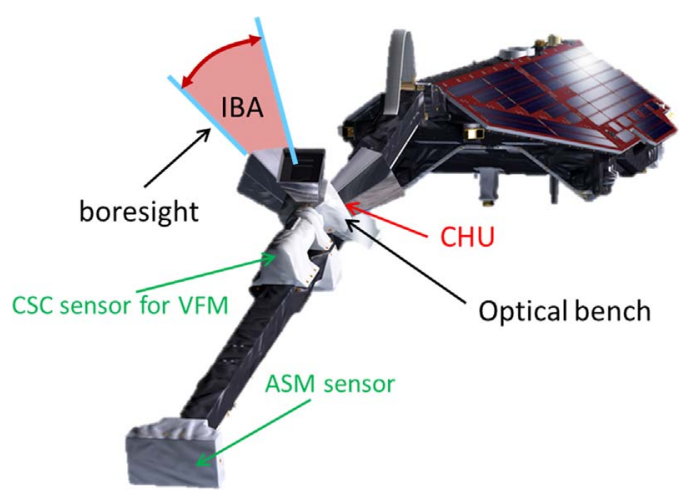

Fig. 2. Inter Boresight Angle (IBA).

\subsection{Corrections applied to the SWARM attitude observations - astronomical aberration}

Astronomical aberration is the shift in the apparent direction of the line of sight to an infinitely distant target, due to the vector combination of the velocity of light and the velocity of the observer relative to the inertial coordinate system (J2000.0). The $\mu$ ASC attitude data can be corrected for astronomical aberration on-board if provided with the spacecraft velocity information. However, since the Swarm velocity information is not immediately available to the $\mu \mathrm{ASC}$, correction for astronomical aberration is applied during post processing on ground. This is performed using the total observer velocity; the velocity of Earth around the Sun and the velocity of the Swarm spacecraft in their orbit around the Earth, as reported by the GPS measurements.

\section{Modelling of the thermo-elastic effects}

\subsection{Thermo-elastic effects of optical bench}

The effect of the astronomical aberration (the difference between blue and red curve in Fig. 3) has a major impact on the IBAs. The aberration correction is not implemented on-board and is therefore fully applied in the first step of the on-ground attitude post processing (contrary to claim of [1]). The description of the algorithms for the Swarm data ground processing specification can be found in [4]. A relative comparison of the aberration corrected attitudes with uncorrected ones shows a significant improvement in stability of the CHU (see Fig. 3 for Swarm Alpha results). The RMS of the IBA of the first CHU pair (CHU A - CHU B) pair is reduced by significant 13.5 arcseconds for the Swarm Alpha as well as Bravo, while the improvement for Swarm Charlie is 11.4" (Table 3). A similar improvement is visible in the other two IBA pairs (CHU A - CHU C and CHU B - CHU C), where the RMS is reduced by 10.4" (Swarm Bravo, IBA pair 3, CHU B - CHU C) and 14.8" (Swarm Alpha, IBA pair 2, CHU A - CHU C) as seen from Table 3 . Considering the first step of attitude correction, 


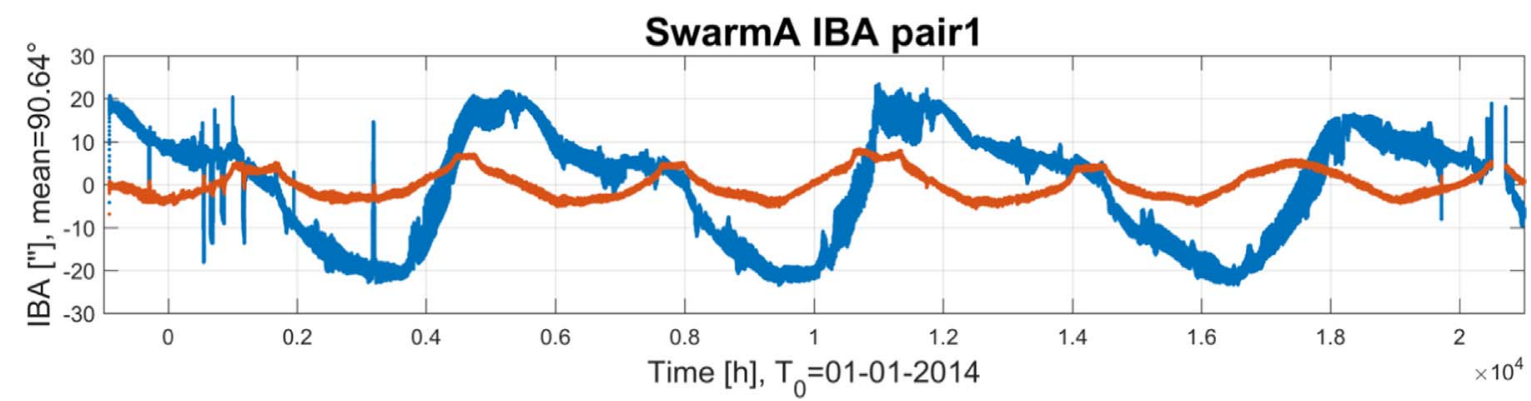

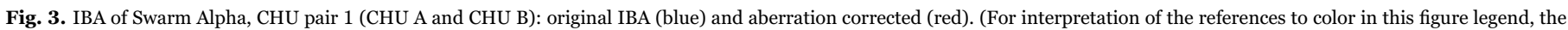
reader is referred to the web version of this article.)

Table 2

Thermal model output coefficients for Swarm Alpha.

\begin{tabular}{|c|c|c|c|c|}
\hline & Alpha & Beta & Gamma & \\
\hline \multicolumn{5}{|l|}{ CHU A } \\
\hline Constant & $-4.67067 \mathrm{e}-01$ & $+4.59718 \mathrm{e}-01$ & $+2.36543 \mathrm{e}-01$ & {$\left[{ }^{\prime \prime}\right]$} \\
\hline $\mathrm{T}_{\text {CHU_A }}$ & $+3.58190 \mathrm{e}-03$ & $-3.52161 \mathrm{e}-03$ & $-7.31744 \mathrm{e}-01$ & {$\left[{ }^{\prime \prime} /{ }^{\circ} \mathrm{C}\right]$} \\
\hline $\mathrm{T}_{029}-\mathrm{T}_{\mathrm{CHU} \_\mathrm{A}}$ & $-9.04363 e-03$ & $+8.89788 \mathrm{e}-03$ & $+4.50041 \mathrm{e}-01$ & {$\left[{ }^{\prime \prime} /{ }^{\circ} \mathrm{C}\right]$} \\
\hline $\mathrm{T}_{032}-\mathrm{T}_{\mathrm{CHU} \_\mathrm{A}}$ & $-1.04154 \mathrm{e}-01$ & $+1.02497 \mathrm{e}-01$ & $-1.12265 \mathrm{e}+00$ & {$\left[{ }^{\prime \prime} /{ }^{\circ} \mathrm{C}\right]$} \\
\hline \multicolumn{5}{|l|}{ CHU B } \\
\hline Constant & $-1.19000 \mathrm{e}+00$ & $+2.03750 \mathrm{e}+00$ & $+5.51564 \mathrm{e}+00$ & {$\left[{ }^{\prime \prime}\right]$} \\
\hline $\mathrm{T}_{\mathrm{CHU} B \mathrm{~B}}$ & $+3.27044 \mathrm{e}-01$ & $-4.03134 \mathrm{e}-01$ & $-3.12725 \mathrm{e}-02$ & {$\left[{ }^{\prime \prime} /{ }^{\circ} \mathrm{C}\right]$} \\
\hline $\mathrm{T}_{029}-\mathrm{T}_{\mathrm{CHU} \_\mathrm{B}}$ & $-2.10603 \mathrm{e}-01$ & $+4.04762 \mathrm{e}-02$ & $-7.31304 \mathrm{e}-01$ & {$\left[{ }^{\prime \prime} /{ }^{\circ} \mathrm{C}\right]$} \\
\hline $\mathrm{T}_{032}-\mathrm{T}_{\mathrm{CHU} \_\mathrm{B}}$ & $+2.40148 \mathrm{e}-01$ & $+6.57657 \mathrm{e}-02$ & $+2.07894 \mathrm{e}+00$ & {$\left[{ }^{\prime \prime} /{ }^{\circ} \mathrm{C}\right]$} \\
\hline \multicolumn{5}{|l|}{ CHU C } \\
\hline Constant & $+4.08584 \mathrm{e}-02$ & $+2.99157 \mathrm{e}-02$ & $+1.17996 \mathrm{e}+01$ & {$\left[{ }^{\prime \prime}\right]$} \\
\hline $\mathrm{T}_{\text {CHU_C }}$ & $+1.36145 \mathrm{e}-02$ & $+9.95873 \mathrm{e}-03$ & $-2.01007 \mathrm{e}-01$ & {$\left[{ }^{\prime \prime} /{ }^{\circ} \mathrm{C}\right]$} \\
\hline $\mathrm{T}_{029}-\mathrm{T}_{\text {CHU_C }}$ & $+1.57687 \mathrm{e}-02$ & $+1.15304 \mathrm{e}-02$ & $+4.97267 \mathrm{e}-01$ & {$\left[{ }^{\prime \prime} /{ }^{\circ} \mathrm{C}\right]$} \\
\hline $\mathrm{T}_{032}-\mathrm{T}_{\mathrm{CHU} \_\mathrm{C}}$ & $+8.06663 \mathrm{e}-03$ & $+5.89875 \mathrm{e}-03$ & $+2.25162 \mathrm{e}+00$ & {$\left[{ }^{\prime \prime} /{ }^{\circ} \mathrm{C}\right]$} \\
\hline
\end{tabular}

Table 3

RMS for the IBA angles solutions (original, aberration corrected and temperature corrected).

\begin{tabular}{llll}
\hline & Swarm Alpha & Swarm Bravo & Swarm Charlie \\
\hline IBA 1 (A-B) & & & \\
Original & 17.1119 & 17.2697 & 17.8227 \\
Abr. Corr. & 3.6450 & 3.7011 & 6.4081 \\
Temp. Corr & 2.0655 & 2.6311 & 2.5803 \\
IBA 2 (A-B) & & & \\
Original & 17.0170 & 17.5293 & 17.2528 \\
Abr. Corr. & 2.2115 & 3.5517 & 2.9427 \\
Temp. Corr & 2.1326 & 2.3842 & 2.2745 \\
IBA 3 (A-B) & & & 14.2941 \\
Original & 14.2831 & 14.7592 & 2.5795 \\
Abr. Corr. & 2.7318 & 4.3382 & 2.1924 \\
Temp. Corr & 2.4830 & 2.6460 & \\
\hline
\end{tabular}

where effect of astronomical aberration is removed, a huge improvement in the attitude determination is achieved (Fig. 3).

Since the optical bench should ensure mechanical and thermal stability of the system, the IBA after aberration correction is expected to be constant. Although an exhaustive thermo-mechanical analysis was carried out prior to the launch of Swarm in order to minimize thermal effects on the attitude of the CHU, a small but still significant thermal effect remains in the attitude data (red curve in Fig. 3). This was detected by comparing the IBA between the CHU pairs to temperature data (Fig. 4) on all three Swarm satellites; see the red curve on Fig. 3 for the case of Swarm Alpha. The more than two-year long time series shows periodic variations that are clearly correlated with temperature variations measured on three Swarm satellites (Table 1.). When comparing the IBA of CHU pair 1 (shown on Fig. 3) with selected temperatures extracted from thermistor observations (Fig. 4), conclusion can be drawn that IBA variation of aberration corrected data has thermal signature. The star camera arrangement on the Swarm configuration maximizes the aberration correction effect when the satellite orbit planes are down-dusk oriented, which is also the configuration when the temperature is highest, and minimal in the noon-midnight orbit. This results in identical periods of both the aberration and thermal driven IBA variations, which are corrected onground.

For the purpose of correcting the attitude data for thermal effects, a symmetrical thermal compensation model was determined from an integer (2) number of thermal periods (to reduce potential spectral leakage, e.g. 2 times 268 days for the Swarm Alpha) determined by the right ascension of the ascending node and the Sun angle. Since all three Swarm satellites had orbital maneuvers at the beginning of the mission, the start of the modelling period was selected after the last maneuver (see green dashed lines in Figs. 5, 6 and 7 for the selected periods). The temperatures selected for thermal modelling are those observed by the CHU $\left(T_{1}\right)$ and OB thermistors with codes THT00029 (referred in formulas as $T_{2}$ ) and THT00032 (referred in formulas as $T_{3}$ ), respectively (Figs. 1 and 4). In order to guarantee thermal model's accuracy, only valid $\mathrm{CHU}$ attitudes data were used in the determination of the model parameters. Attitude data that is recorded in high-rate mode, has a big bright object flag or has a confidence level (misfit between the observed and the cataloged stars, expressed in unit that corresponds to arc-seconds) of more than 5 was not used in modelling.

To analyze the relative misalignment of the three CHU's, a fixed frame was defined by the most stable axes of the three CHU frames. The primary requirement of the fixed frame was a minimum dependancy on the observed thermal effects to ensure a frame free from any deformation. For that purpose, we analyzed the relative misalignments of each $\mathrm{CHU}$, mounted on the $\mathrm{OB}$ due to temperature variations. The fixed frame is then defined such that the Z-axis coincides with the camera optical axis and the $\mathrm{X}$ and $\mathrm{Y}$ axes are in the plane of the CCD, with the $\mathrm{X}$-axis pointing horizontally to right hand side and $\mathrm{Y}$ axis pointing up to complete an orthogonal right-handed coordinate system. Comparing relative misalignments of all three $\mathrm{CHU}$ axes, it was concluded that the plane formed by the bore-sight axes ( $\mathrm{Z}$ axis) of CHU A and C is very stable compared to the other axes and planes. Therefore, the $Z_{F}$ axis of the fixed reference frame is defined as the normal to this plane.

$Z_{F}=\frac{Z_{A} \times Z_{C}}{\left|Z_{A} \times Z_{C}\right|}$

The rotation of the fixed reference frame about the $Z_{F}$ axis is limited by taking an average of both $\mathrm{CHU}$ boresight axes, describing the $X_{F}$ axis.

$X_{F}=\frac{\frac{Z_{A}+Z_{C}}{2}}{\left|\frac{Z_{A}+Z_{C}}{2}\right|}$

The $Y_{F}$ is constructed to complete an orthogonal right-handed coordinate system.

$Y_{F}=\frac{Z_{F} \times X_{F}}{\left|Z_{F} \times X_{F}\right|}$ 

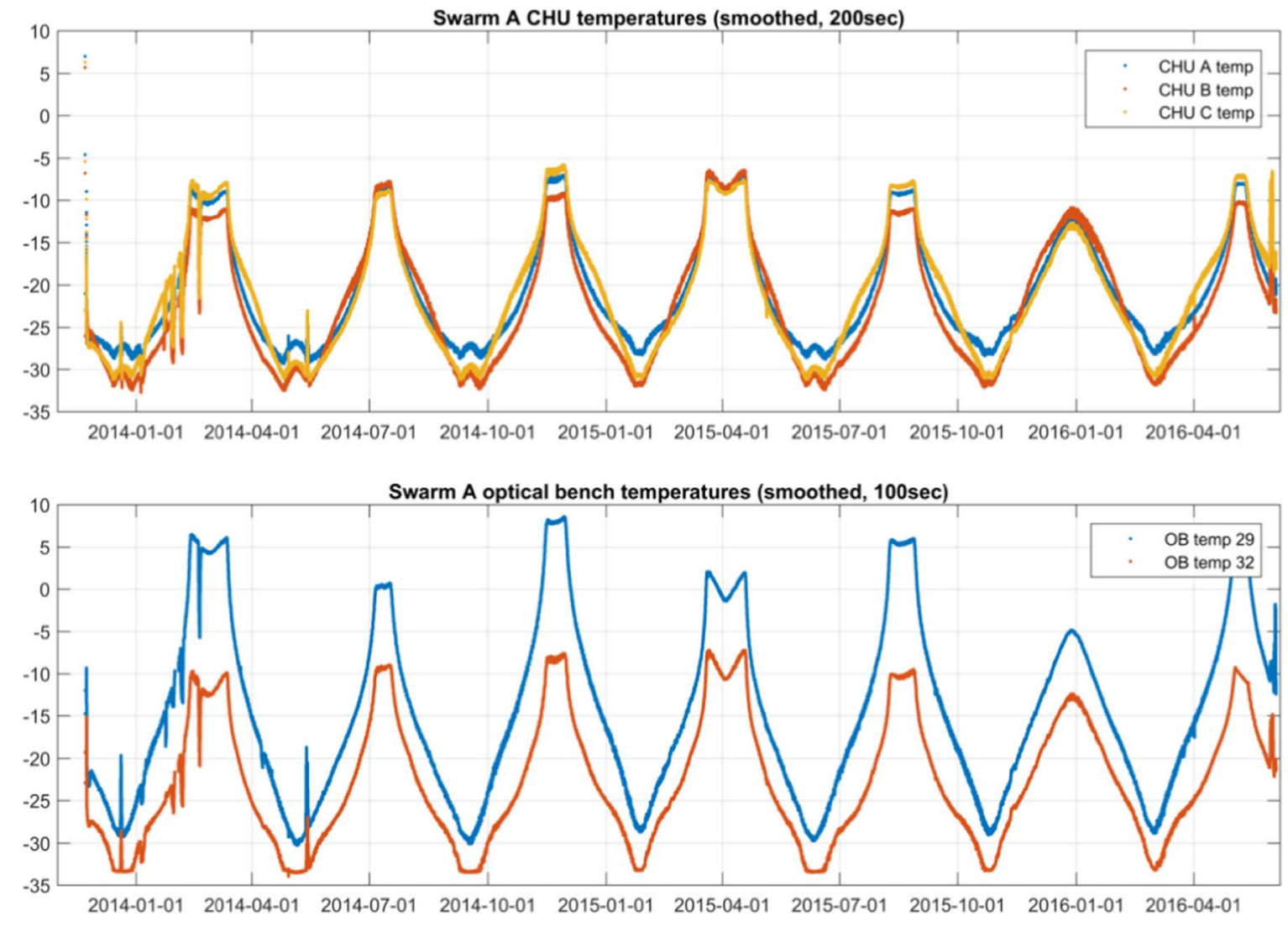

Fig. 4. Swarm Alpha CHU (top) and Optical bench boom (bottom) temperatures.

The subscript letters A, B and C refers to the naming of the CHU.

Rotation of each CHU axis depending on the observed temperature is then estimated relative to fixed frame. The thermal model is then found by Singular Value Decomposition (SVD) fitting of IBA orbital averages and observed temperatures, and its parameters describe how each CHU moves relative to its pre-flight calibrated frame due to the thermal gradients. It is defined as:

$R_{\text {CHUcorrected }}=R_{3}(\gamma) R_{2}(\beta) R_{1}(\alpha) R_{C H U}$

where each rotation is described by:

$R_{1}(\alpha)=\left[\begin{array}{ccc}1 & 0 & 0 \\ 0 & \cos (\alpha) & \sin (\alpha) \\ 0 & -\sin (\alpha) & \cos (\alpha)\end{array}\right]$

$R_{2}(\beta)=\left[\begin{array}{ccc}\cos (\beta) & 0 & -\sin (\beta) \\ 0 & 1 & 0 \\ \sin (\beta) & 0 & \cos (\beta)\end{array}\right]$

$R_{3}(\gamma)=\left[\begin{array}{ccc}\cos (\gamma) & \sin (\gamma) & 0 \\ -\sin (\gamma) & \cos (\gamma) & 0 \\ 0 & 0 & 1\end{array}\right]$

And individual rotation angles are defined as:

$\alpha=\alpha_{0}+\alpha_{1} T_{1}+a_{2}\left(T_{2}-T_{1}\right)+\alpha_{3}\left(T_{3}-T_{1}\right)$

$\beta=\beta_{0}+\beta_{1} T_{1}+\beta_{2}\left(T_{2}-T_{1}\right)+\beta_{3}\left(T_{3}-T_{1}\right)$

$\gamma=\gamma_{0}+\gamma_{1} T_{1}+\gamma_{2}\left(T_{2}-T_{1}\right)+\gamma_{3}\left(T_{3}-T_{1}\right)$

where:

$$
\begin{aligned}
& \mathrm{T}_{1}=\mathrm{T}_{\text {CHU_i }}(\mathrm{i}=\mathrm{A}, \mathrm{B}, \mathrm{C}) \\
& \mathrm{T}_{2}=\mathrm{T}_{\mathrm{THH} \text { Ho029 }} \\
& \mathrm{T}_{3}=\mathrm{T}_{\mathrm{THT00032}}
\end{aligned}
$$

The resulting thermal model with modelling coefficients for Swarm Alpha is shown in Table 2. In the same manner, the models were created for the other two Swarm satellites, which coefficients can be accessed in the electronic supplement.

\section{Results}

The resulting thermal model is applied on each observed attitude of all CHU's for the complete available Swarm dataset (from November 2013 to June 2016).

Since IBA is a valid relative comparison of the CHU stability, we limit our further analysis to the IBA only. This choice is governed by the accuracy of measured orientation, which is the highest in camera pointing direction.

\subsection{IBA analysis - relative comparison of the CHU stability}

After applying thermal correction to the attitude observations, the comparison is made with uncorrected data. Figs. 5, 6 and 7 show these comparisons for each of the three Swarm satellites Alpha, Bravo and Charlie respectively, where green dashed line marks the period from which model parameters are calculated, i.e. from 16-June-2014 to 03Dec-2015.

It is easy to see in these figures, that the smallest correction is applied to the second IBA pair (CHU A - CHU C) on all three Swarm satellites. The reason for this lies in the model definition (described in the previous section), where $\mathrm{Z}_{\mathrm{F}}$ axis of the fixed reference frame is defined as a normal to the plane containing bore-sight axis of CHU A and $\mathrm{CHU} \mathrm{C}\left(Z_{A} \times Z_{C}\right)$. Comparison of relative stabilities shows that these axes are more stable than bore-sight axis of CHU B $\left(Z_{B}\right)$.

The results for all three satellites show the largest correction (Table 3 and plots on Figs. 5, 6 and 7) for the first (CHU A - CHU B) and the third ( $\mathrm{CHU} \mathrm{B}-\mathrm{CHU} \mathrm{C}$ ) IBA pair. It is worth mentioning 

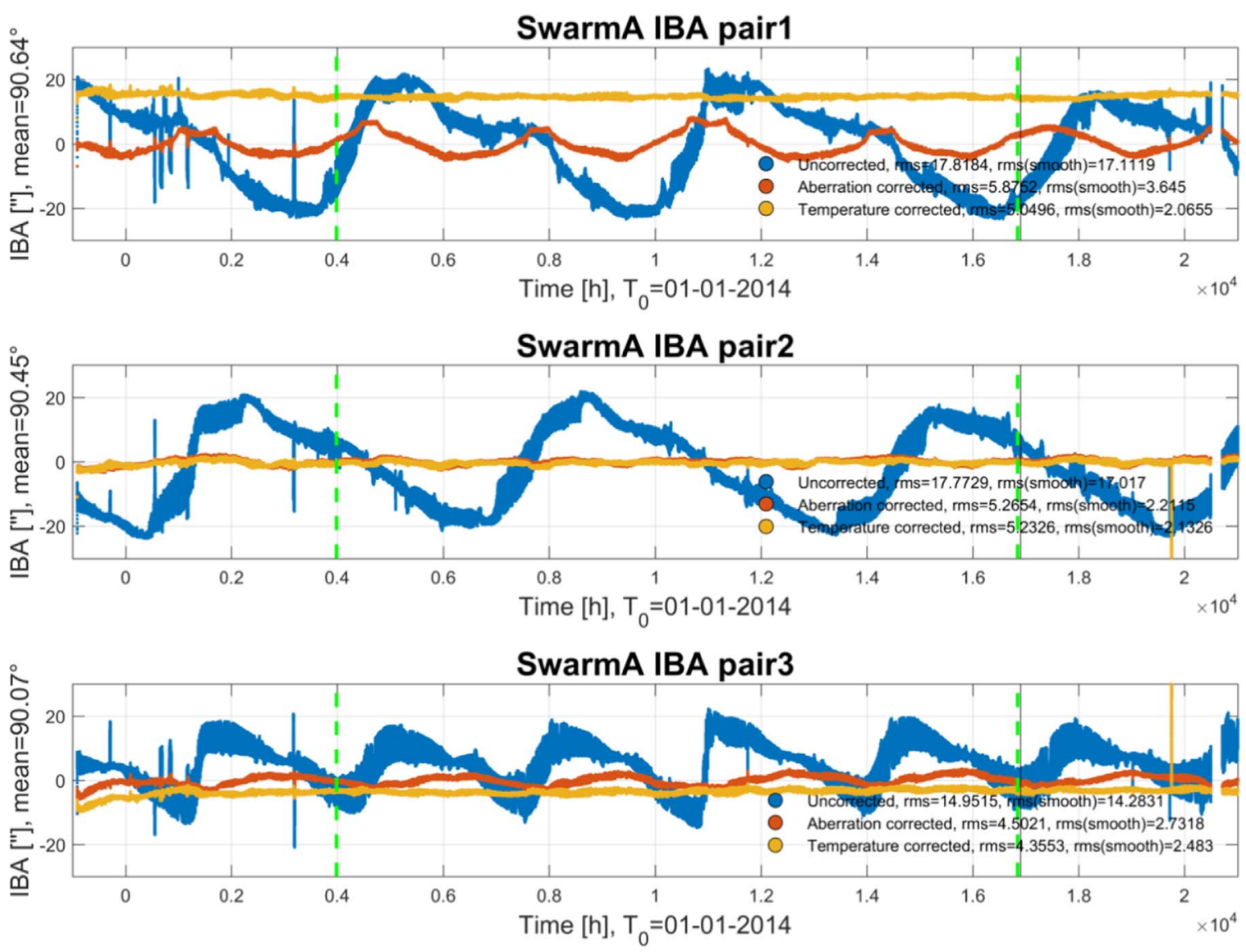

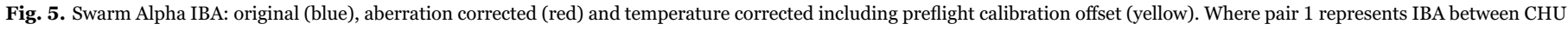

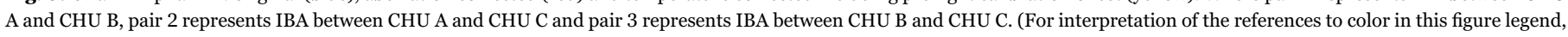
the reader is referred to the web version of this article.)

that both of these IBA pairs have CHU B in common. Considering the high correction applied to the first and third IBA (the first and third plot on Figs. 5, 6 and 7), we can conclude that the main correction is involved in relative orientation where CHU B is involved. This implies that the reason for thermal sensitivity of CHU B can probably be found in its placement on the OB. The placement of the CHU on the edge of the OB may produce such thermal effects.

In this study, we use RMS value of the IBA data for flatness assessment. Values shown on Figs. 5, 6 and 7 show RMS values of the complete dataset and RMS of the smoothed dataset with $200 \mathrm{~s}$ window width to suppress short term noise sources.

The RMS of the first IBA pair (CHU A - CHU B) dataset is reduced from 3.6 to $2.1^{\prime \prime}$, from 3.7 to $2.6^{\prime \prime}$ and from 6.4 to 2.6" for Swarm Alpha, Bravo and Charlie, respectively. The RMS of the third IBA dataset (CHU B and CHU C) is reduced from 2.7 to $2.5^{\prime \prime}$, from 4.3 to 2.6" and from 2.6 to 2.2" respectively for Swarm Alpha, Bravo and Charlie, respectively.

In the case of the most stable CHU combination, i.e. the second IBA pair, the effect of the correction on the IBA is very small but still significant, ranging from 0.1" (for the Swarm Alpha) to 1.2" correction to Swarm Bravo (Table 3).

If one focuses only on the yellow curves on Figs. 5, 6 and 7, and statistics of temperature corrected data on Table 3, the results show small RMS decrease for Swarm Alpha, IBA pair $2\left(0.1^{\prime \prime}\right)$ and significant decrease for Swarm Charlie, IBA pair 1 (3.9'). Significant RMS decrease is also present in all IBA pairs for three Swarm satellites (Table 3), which leaves corrected data smooth and clean from any thermally induced phenomenon. This is especially notable for the corrected data outside of the modelling period (green dashed line on Figs. 5, 6 and 7), which shows excellent results when predicting thermal effects.
The technique presented here shows significant improvement in attitude determination which, after thermal correction, meets the $2^{\prime \prime}$ requirements of Swarm satellite mission [5]. Considering that IBA statistics presented in Table 3 contain measurements of two cameras, the RMS per instrument is assumed to be less than $1 / \sqrt{2}$ times value presented in the table.

Further demonstration of thermal model functionality would be to intentionally modify OB temperature in the same (seasonal) manner, and compensating the effect by applying thermal model. A similar test was performed in the period from 30 May through 3 June 2016, when an investigation of the residuals between Swarm ASM and VFM magnetometer data was conducted on Swarm Alpha. The investigation was performed using two heaters located on the OB (an operational heater $(21 \mathrm{~A})$ mounted on the radiator plate of the $\mathrm{OB}$, and a powerful survival heater $(21 \mathrm{~W})$ located on the OB interface bracket).

Although a heat flow initiated by the OB heaters will obviously be different from the one caused by the Sun, an additional demonstration of the model performance can be made.

\subsection{Heater tests for ASM-VFM residual investigation on Swarm Alpha}

To confirm the validity of the computed thermal model, the data from the heater test ASM-VFM residual investigation, performed on Swarm Alpha, was used.

The first part of the heater test to support the ASM-VFM residual investigation was started on 30.05.2016, when the heater $21 \mathrm{~A}$ mounted on $\mathrm{OB}$ was activated. Before the test start, measured temperature on the $\mathrm{OB}$ was around $-10{ }^{\circ} \mathrm{C}$ for the thermistor THT00029 $\left(T_{2}\right)$, and around $-20.5^{\circ} \mathrm{C}$ on the thermistor THT00032 

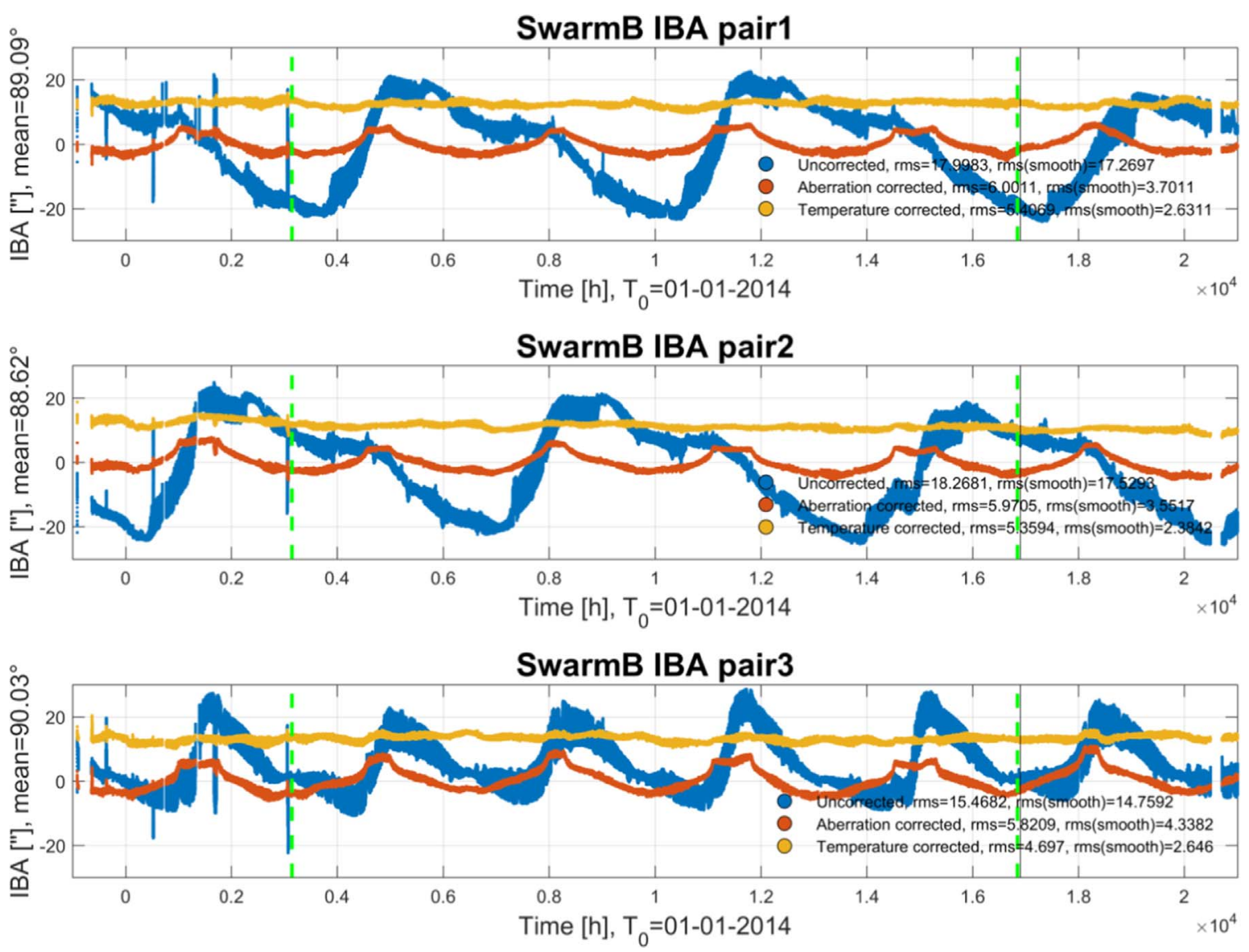

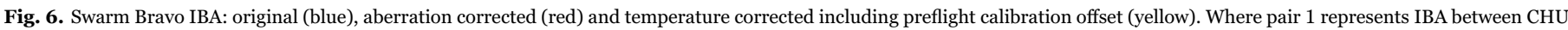

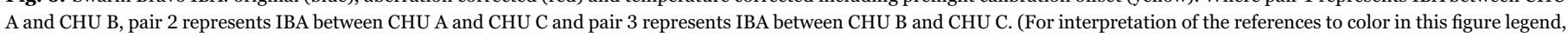
the reader is referred to the web version of this article.)

$\left(T_{3}\right)$, see Fig. 8. Briefly after the test start (07:19:06 UTC) the temperature was increased slowly until it reached a stable balance close to the $-15^{\circ} \mathrm{C}$ at around 22:00 UTC (see red curve in bottom plot of the Fig. 8). The temperature was controlled by the heater until 01.06.2106 at 12:24:40 UTC, when the original temperature was restored.

In the second part of the test, which started on 02.06.2016., the heater $21 \mathrm{~W}$ on $\mathrm{OB}$ was activated. Before the test, the temperature measured by thermistor THT00029 was around $-13{ }^{\circ} \mathrm{C}$ and had almost returned to its expected value after the previous test with heater $21 \mathrm{~A}$ (see blue curve in the bottom plot of the Fig. 8). At 13:17:52 UTC, the new temperature range from $-7{ }^{\circ} \mathrm{C}$ to $-9{ }^{\circ} \mathrm{C}$ was achieved by setting up the heater control loop. After $17 \mathrm{~h}$, at 06:22:02 UTC at 03.06.2016., control temperatures were increased to $-4{ }^{\circ} \mathrm{C} /-2{ }^{\circ} \mathrm{C} .8 \mathrm{~h}$ after (at 14:12:23 UTC), the control loop was disabled and the original settings were restored (blue curve in the bottom plot of the Fig. 8).

When temperatures during the heater tests (Fig. 8) are compared with the observed IBA's on Swarm Alpha (Fig. 9), a correlation can be seen in the test when heater $21 \mathrm{~A}$ was activated. It is noticeable that CHU and THT00032 thermistors are very sensitive to the temperature variation of the heater $21 \mathrm{~A}$ (top plot of Fig. 8 and red curve in the bottom plot of Fig. 8). However, this is not the case with variation of temperature by the heater $21 \mathrm{~W}$, where temperature variation of that heater affects only thermistor THT00029 (blue curve on the bottom plot if the Fig. 8). This is probably due to the different location of the heaters, which causes a different heat flow.

When the thermal model is applied to the heater test data, the results show an interesting behavior. Firstly, it is noticeable how activating the $21 \mathrm{~W}$ heater in the second test does not change significantly the IBA or the observed CHU and THT0032 temperatures.
However, it does affect the temperature measured on thermistor THT00029. Due to the fact that temperature observed by thermistor THT0029 affects only the third (gradient) member in formula 7 , the correction of the IBA is very small and the yellow curve showing the corrected IBA stays almost flat (Fig. 9).

The most significant impact on IBA is seen in the first IBA pair in of the first test (top panel in the Fig. 9), where the model over-compensate the temperature effect. This is expected, since heat generated in these tests originates from different source than the Sun, which are implemented in our thermal model and therefore cause a different heat flow.

The presented results show that the model, even though not tailored to this type of heat variation, behaves excellent and removes the largest part of the thermally induced OB instabilities that cause IBA variations.

\subsection{Sources of remaining uncertainties}

By the virtue of the silicon carbide-carbon fiber optical bench design, absolute temperatures of the system are unknown. Therefore, the proxy temperatures and temperature gradients used in thermal modelling, observed by limited number of thermistors, could introduce a residual error in modelled attitude of around $0.5^{\prime \prime}$. The results also contain errors $\left(\ll 0.5^{\prime \prime}\right)$ due to the singular value decomposition imperfect fitting and in-orbit thermal effects not captured by the orbital averages modelling technique. Furthermore, the model may leave corrected attitudes with unresolved signals of no thermal origin.

However, all of the effects combined show that the residual signal is smaller than $+/-1^{\prime \prime}$, which is well below the mission requirement of $2^{\prime \prime}$. 

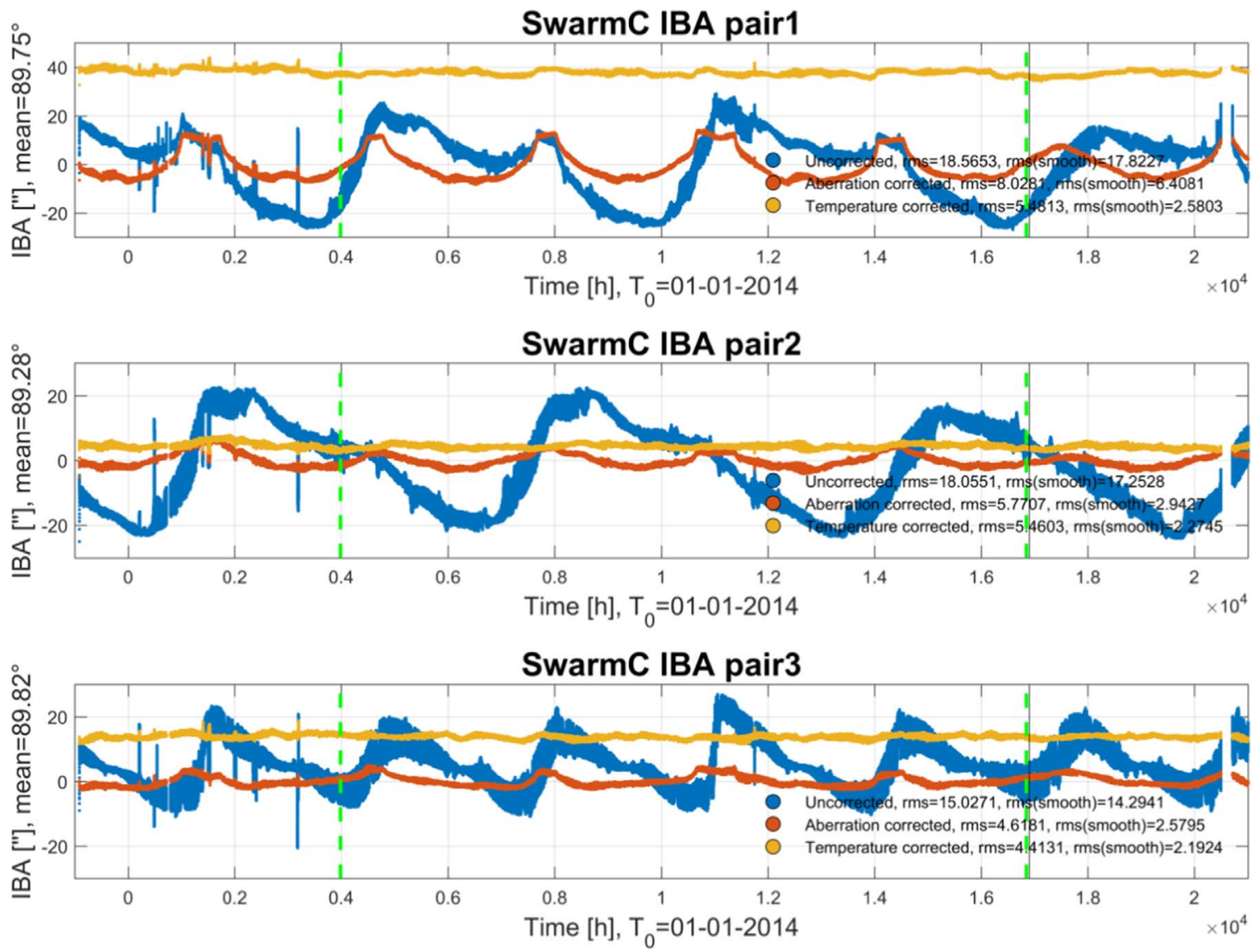

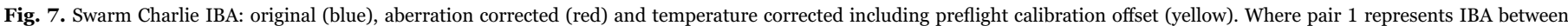

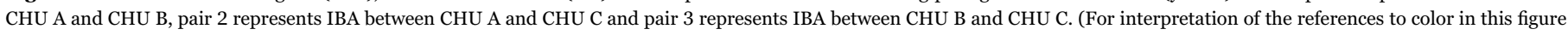
legend, the reader is referred to the web version of this article.)
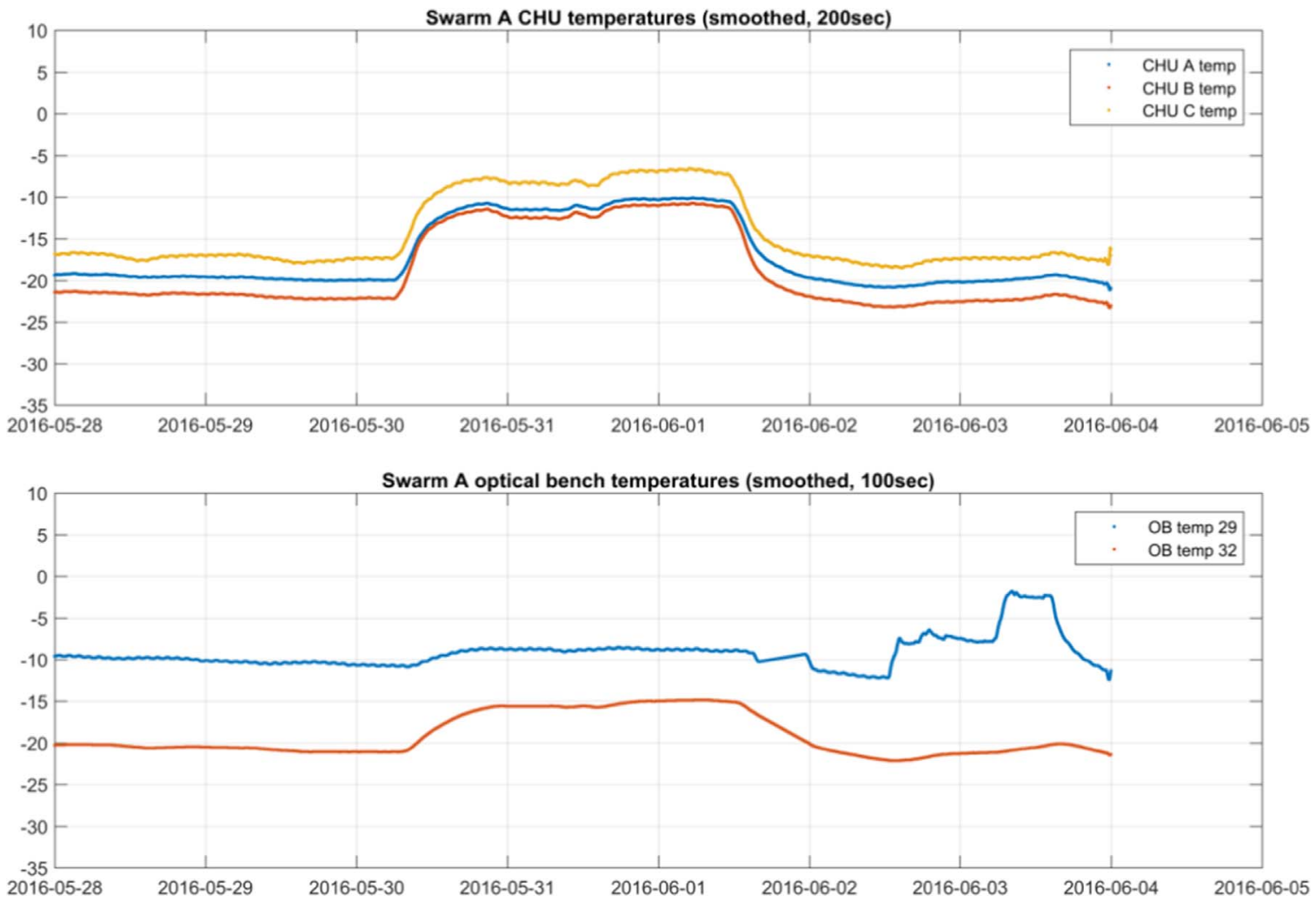

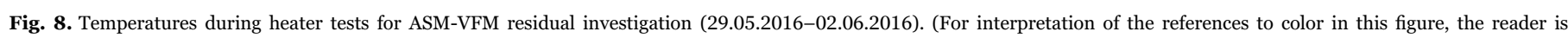
referred to the web version of this article.) 

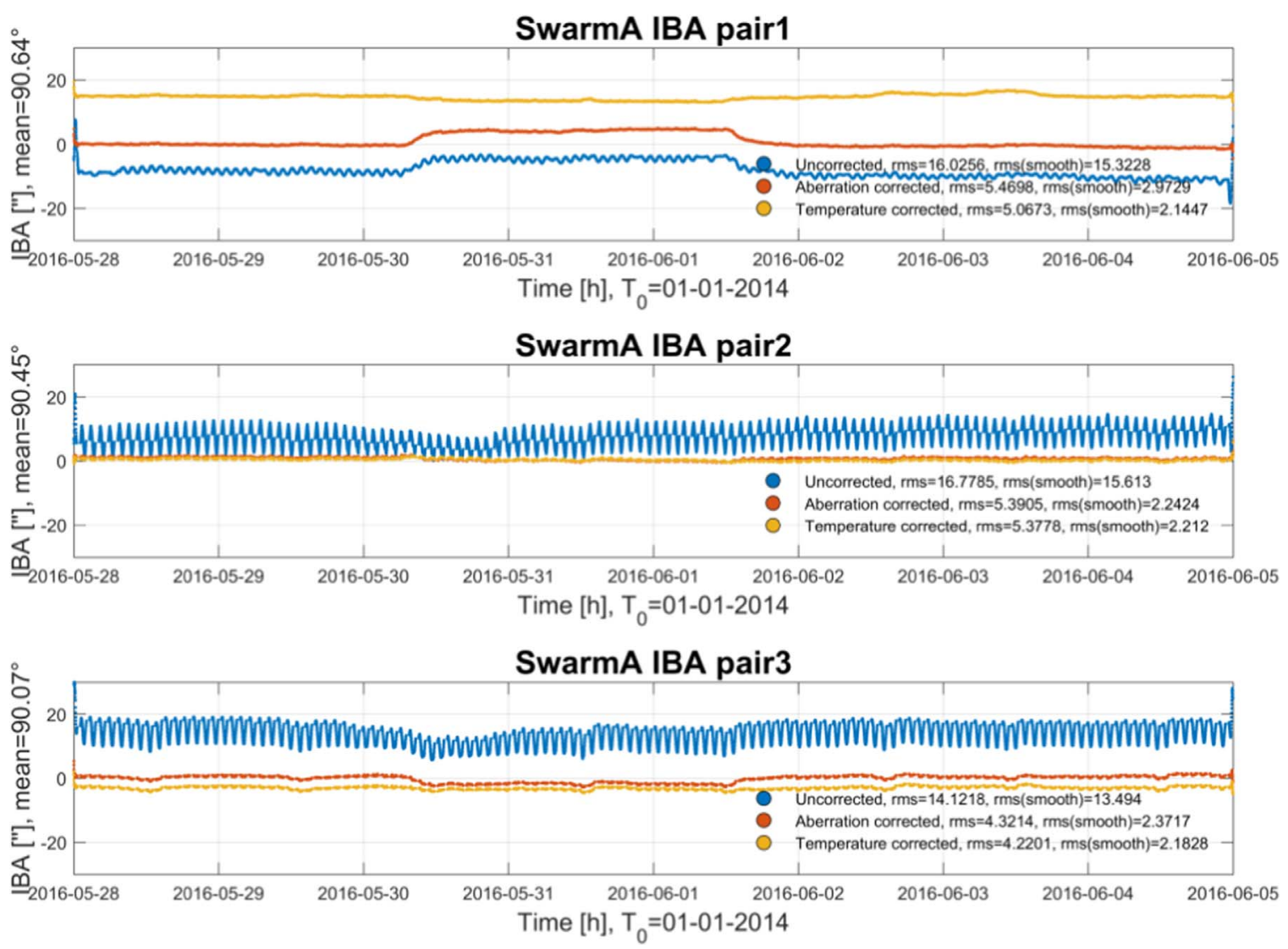

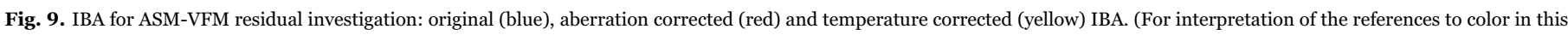
figure legend, the reader is referred to the web version of this article.)

\section{Conclusions}

In this paper, periodical variations in relative orientation between the Swarm CHU are discussed and a model for their correction in the Swarm data processing is presented.

Since the observations obtained by $\mu$ ASC are affected by the astronomical aberration, the corresponding correction is applied in the on-ground processing. Considering the mechanically and thermally stable optical bench, the IBA after aberration correction is expected to be in the $1^{\prime \prime}$ range. However, after correction for the aberration effect, a small but still significant thermal signal remains in the IBA data, which clearly correlates with temperature variations measured on three Swarm satellites. Therefore, a conclusion is drawn that IBA variation of aberration corrected data has thermal signature.

We present a model for the correction of the thermo-elastic instabilities of the Swarm OB, which cause misalignments between the CHU relative orientations. The model is based on two full periods of Swarm orbital plane to the Sun angle, which is recognized as the major driving force of thermal instabilities, and is computed by Singular Value Decomposition fitting of IBA orbital averages and observed temperatures. The improvement of attitude determination accuracy and CHU stability was analyzed by relative comparison of the three IBA's on the three Swarm satellites, Alpha Bravo and Charlie. The results after applying thermal corrections show a small decrease in RMS for Swarm Alpha IBA pair $2\left(0.1^{\prime \prime}\right)$ and significant decrease for Swarm Charlie IBA pair $1\left(3.9^{\prime \prime}\right)$. In the case of the most stable CHU's combination, the second IBA pair, the correction is very small but still significant, ranging from $0.1^{\prime \prime}$ (for the Swarm Alpha) to 1.2" correction for Swarm Bravo. Overall, corrected attitude data is very smooth and clean from any thermally induced phenomenon. This is especially notable for the corrected data outside of the modelling period, which shows excellent results when predicting thermal effects. Therefore, the technique presented here shows an improvement in attitude determination that, after correction, meets the 2 " requirements of Swarm satellite mission.

Furthermore, the robustness and validity of the thermal model was demonstrated using data collected during a test in support of the ASMVFM residual investigation on Swarm Alpha, which was performed in the period from 30 May through 3 June 2016. The investigation was performed using two heaters located on $\mathrm{OB}$ (an operational heater mounted on the radiator plate of the $\mathrm{OB}$, and a powerful survival heater located on the $\mathrm{OB}$ interface bracket). Results show that the thermal model slightly over-compensates the temperature effect on the first IBA, but fully corrects the effect on the other two IBA.

The critical finding from these tests is that thermal model, even though not tailored to this specific type of heat flow (where heat sources are heaters and not the Sun) behaves excellent and successfully removes the thermally induced OB instabilities. Consequently, the study demonstrates the importance of the OB pre-launch analysis and test, which may ensure minimum thermal gradient on satellite optical system and therefore maximum attitude accuracy and stability between the cameras.

In [1] an assumption of a perfect optical bench system and/or a flawed aberration correction is used to explain the observed IBA variations. The analysis and thermal model presented herein, shows that aberration correction is performed correctly. Instead, the origin of the IBA variations is a thermal gradient and the effect is fully recoverable in the ground processing by a simple thermal model.

The presented model is now being implemented in the Swarm data processing. 


\section{References}

[1] S. Maus, Mysterious misalignments between geomagnetic and stellar reference frames seen in CHAMP and Swarm satellite measurement, Geophys. J. Int. 203 (2015) 1873-1876.

[2] P.S. Jørgensen, J.L. Jørgensen, T. Denver, $\mu$ Asc: a miniature star tracker, in Proceedings of The 4S Symposium: Small Satellites, Systems and Services (ESA SP571), 20-24 September 2004, La Rochelle, France, Editor: B. Warmbein. Published on CDROM., id.55.1, 2004ESASP.571E.55J, 2004.
[3] C. Pereira, I. Rathband, The composite tube assembly of Swarm, in: Proceedings of the 12th European Conference on Spacecraft Structures, Materials \& Environmental Testing, ESA/ESTEC, Noordwijk, The Netherlands, March 20-23, 2012, SP-691, 2012.

[4] L. Tøffner-Clausen, P.E. Holmdahl Olsen, Swarm Level 1b Processor Algorithms, SW-RS-DSC-SY-0002, National Space Institute, Technical University of Denmark, 2015.

[5] R. Haagmans, Swarm: The Earth's Magnetic Field and Environment Explorers Mission Requirements Document for Phases B, C/D, E1, 2005. 